\title{
DESIGN AND IMPLEMENTATION OF COORDINATION PROTOCOLS FOR DISTRIBUTED COOPERATING OBJECTS - A GENERAL GRAPH-BASED TECHNIQUE APPLIED TO CORBA
}

\author{
Khalil DRIRA \\ and Frédéric GOUËZEC \\ and Michel DIAZ
}

LAAS-CNRS, 7 Av. Colonel Roche 31077 Toulouse Cedex FRANCE drira@laas.fr, gouezec@laas.fr, diaz@laas.fr

\begin{abstract}
This paper introduces a technique for the design and the implementation of coordination protocols in distributed cooperative applications using distributed objects and CORBA. An application architecture is designed as a group of cooperating "sites" having symmetrical configurations represented using "The Coordination Graph", a graph structure whose nodes may be associated with abstract software components acting according to user-defined behaviors. The graph structure may be transformed following a set of rewrite rules. Application of a rule leads to the deletion and creation (at each site) of cooperating objects associated respectively with the nodes being removed from or inserted into the graph. These main coordination actions allow the configuration of applications to be automatically managed by the coordination service. The transformation rules are also used to control the cooperative activity supported by the application, i.e. for example, managing user access to the shared cooperation information. Hence the proposed technique allows both architectural and behavioral coordination issues to be handled for distributed applications.
\end{abstract}




\section{INTRODUCTION}

The emergence of high speed networks has given rise to a number of real experimentations of multimedia cooperative activities in various industrial environments.

Distribution, concurrency and architecture dynamic evolution are important features for cooperative activities support systems design that traditional software architecture models are inappropriate to describe $[1,10,15]$.

In component-oriented programming techniques [15], managing the architecture dynamic evolution is tantamount to managing components insertion and removal. This is called "configuration management" and constitutes the first interest of our graph-based technique for the description of coordination protocols in distributed cooperative applications. The second interest of this technique is the enabling and the control of the cooperation in these applications.

Specifying the coordination protocol independently of the technique used to describe the coordinated components behaviors is an interesting issue and constitutes an important contribution of coordination models and languages [7]. The coordinated components may cooperate by message exchange using interaction ports when communicating processes are used, via a shared space as for Gamma [4] and Linda based [5] models or by method invocation using a Request Broker for CORBA-based distributed architectures [13].

The technique presented in this paper, uses graphs as the basic formalism for the specification of configurations. This choice takes advantage of the powerfulness of graphs for abstract representation of the different entities and dependencies that may be involved in the coordination description. The coordination graph is defined as a graph whose nodes may be associated with user-defined components implementing cooperating object behavior.

The configuration evolution is described as a set of graph transformation rules. On one hand, a rule specifies the transformation conditions including graph matching (i.e. graph morphism) allowing activity control to be expressed. On the other hand the rule specifies the transformation result including graph structure modification (deleting and creating vertices and edges), and configuration modification (deletion and creation of objects) allowing both the cooperation state and sites architecture to be updated.

This paper is organized as follows: section 2 describes the coordination model underlying our approach. Section 3 describes the application to distributed objects using CORBA. Section 4 discusses a simple example of coordination. Section 5 compares our approach with graph-based coordination approaches. In conclusion we discuss current state of the provided environment and future work. 


\section{THE COORDINATION MODEL}

As depicted by Fig. 1, the constructive model underlying the proposed coordination technique is composed of the following three basic parts obtained by specialization of the model of [6]:

The coordinated entities These are objects, which are specialized in cooperative information production, transformation and exchange.

The coordination laws describe how objects coordinate themselves [6]. The coordination laws are those described by the graph transformation rules in our model.

The coordination medium makes communication among objects possible and, moreover, can serve to aggregate objects that should be manipulated as a whole. Examples are classic media such as semaphores or monitors or channels, or more complex media such as tuple spaces, blackboards, pipelines, and the like [6]. In our model, the coordination medium is the coordination graph structure together with the access protocol over the CORBA broker.

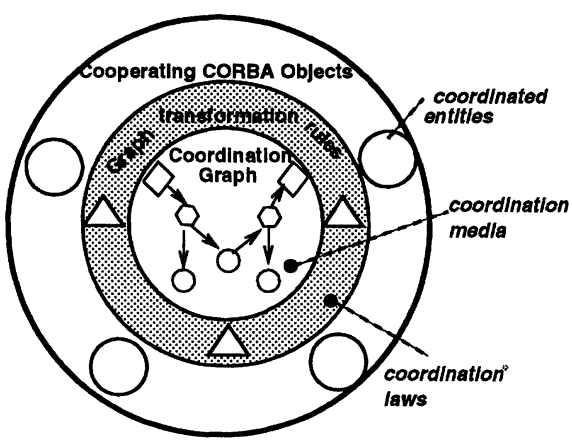

Figure 1 The coordination model with coordination graph

\section{Associated applications architecture}

Applications have the abstract architecture described in Figure 2. Cooperative objects run concurrently on distributed sites and are organized into groups. When no coordination action is involved, cooperative objects may directly communicate with their peers, without using the coordination medium. This is called plain intra-group cooperation. Cooperative Objects use the coordination medium when global actions related to the activity management or to the configuration management are required. 


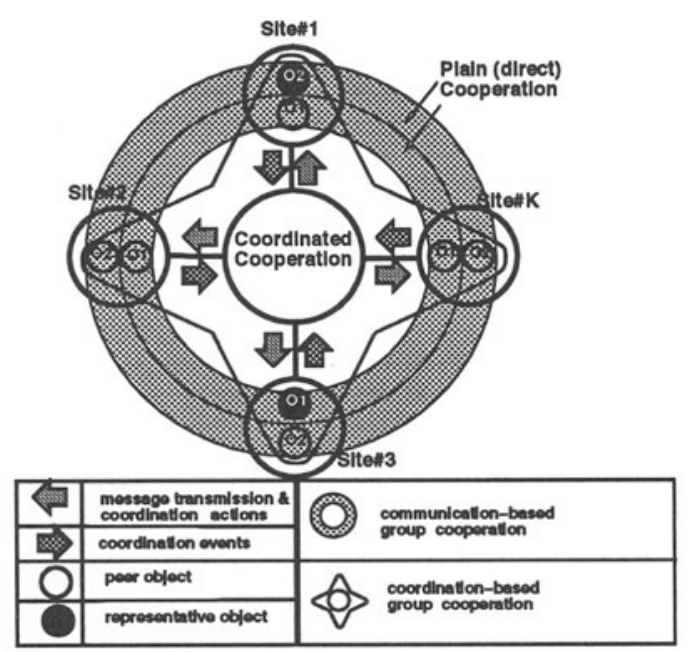

Figure 2 Abstract architecture of applications

\section{The coordination graph}

The coordination graph is a typed graph whose nodes are associated with behaviors of cooperating entities described using object-oriented techniques. Other attributes may be added to nodes. These are user-defined parameters.

The attribute "behavior" associated with graph nodes allows configuration to be represented by the graph and allows this configuration:

- to be managed by actions associated with transformation rules;

- to be initialized for new participants.

For example a graph composed of two nodes: $n_{1}:$ TextWindow $\longrightarrow$ $n_{2}$ : TextWindow leads to the configuration : $O_{1} \| O_{2}$ on each connected site, where $O_{1}$ and $O_{2}$ are two concurrent entities of type TextWindow. Using CORBA, these entities are CORBA objects.

\section{The coordination rules}

The coordination rules are described as transformation rules over the coordination graph. A graph transformation rule is again a graph where we distinguish three fragments: the preserve, the retract and the insert fragments. A guard may also be used in a rule to describe boolean conditions on the variables appearing in the graph.

Graph transformation operates as follows:

- A subgraph isomorphic to the require = preserve $\cup$ retract fragment is searched. 
- If any found, the subgraph matching the retract part of the rule is removed from the original graph. A graph isomorphic to the insert fragment is then embedded into the previously transformed graph.
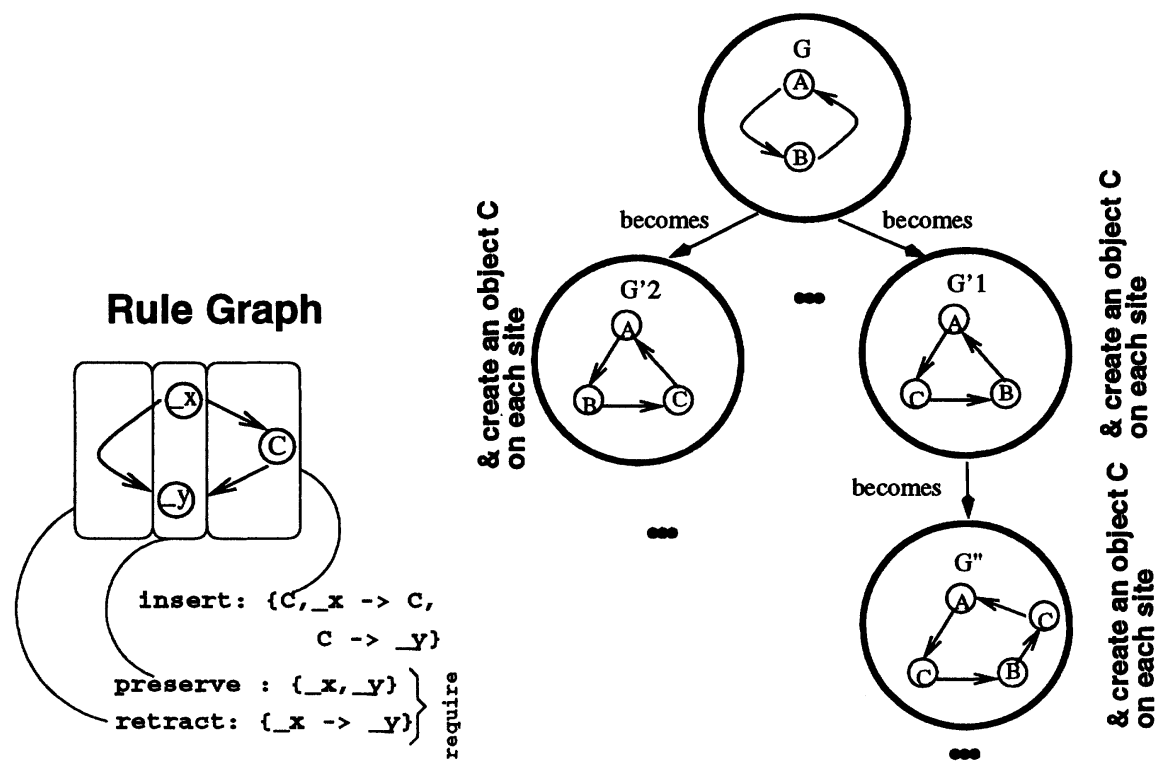

Figure 3 (a) a transformation rule, (b) an initial graph and some applications

A simple graph transformation rule and its application to a graph are shown in Fig. 3.(a) and Fig. 3.(b) respectively. In the transformation " $G \longrightarrow G_{1}^{\prime}$ ", the require fragment is mapped on the original graph by associating:

node $-x$ of the rule with node $A$ of the graph node $-y$ of the rule with node $B$ of the graph edge $-\mathbf{x} \longrightarrow-\mathbf{y}$ of the rule with edge $\mathrm{A} \longrightarrow \mathrm{B}$ of the

graph.

In a coordination graph description, we should be able to classify cooperating entities, e.g. to distinguish a cooperating entity handling text from another handling images. For this, we use a typed version of graph transformation techniques: nodes have types that transformations must preserve.

The applicability of a rule is conditioned by typed graph morphism laws without distinction for runnable nodes.

The application of a rule leads to the graph transformation and the configuration management actions. In the case of the previous example a cooperating entity whose behavior is specified by the node $\mathrm{C}$ is created on each site every time the rule is applied. 


\section{IMPLEMENTATION FOR CORBA-BASED APPLICATIONS}

The implemented environment includes a factory that allows different coordination media to be created simultaneously. Each medium is composed again of two CORBA objects: the coordination server and the communication server. This package is developed using the IDL/Java mapping; it is generic except the Java classes that programmers should write to describe coordination rules. A Cooperating Site object that uses this service may be written using the IDL/Java or IDL/C++ mappings; it includes the cooperating objects behavior and a number of other objects that will be described in the sequel. We also use the CorbaScript environment [14] to easily administrate the system.

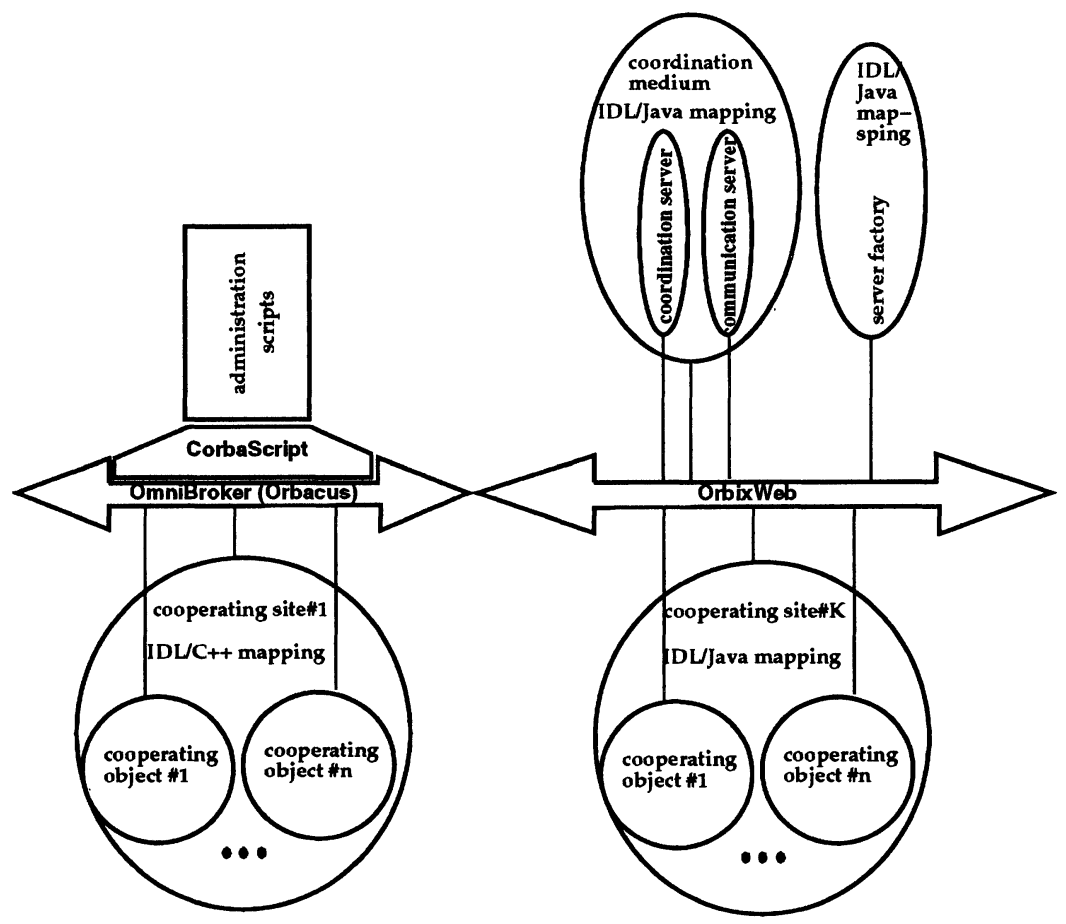

Figure 4 The Corba components involved in an application

The architecture of this service allows the programmer to develop a standalone application or an applet executed using WWW browsers. The communication being relayed by the group communication manager, we are in conformance with the safety requirements of web browsers stating that an applet cannot establish a connection except with the Web server from where it was downloaded. 


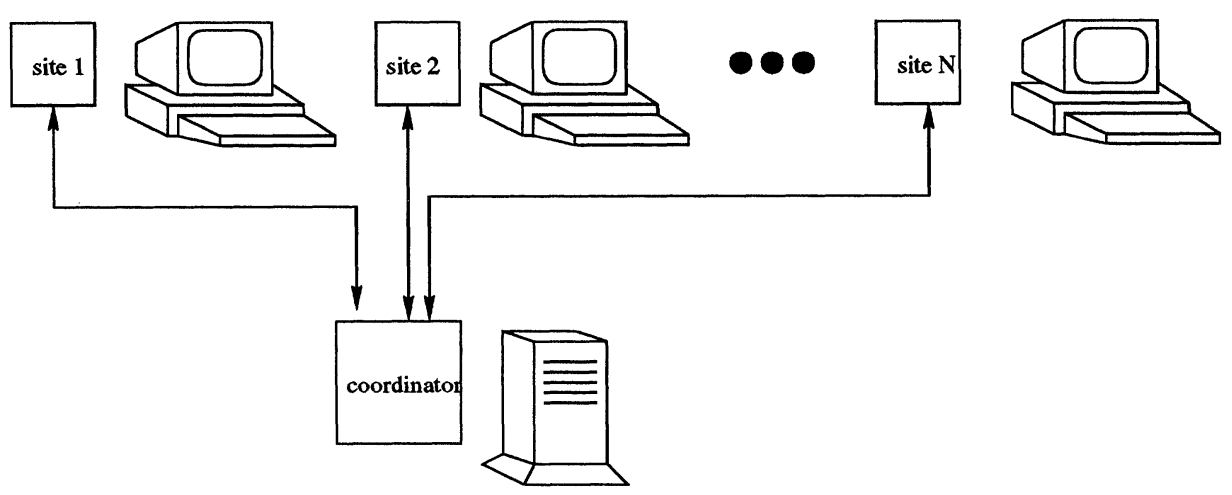

Figure 5 Logical applications topology distinguishing communication paths

As depicted in Fig. 5, a coordination medium that runs on the same machine as the Web-server allows Cooperating objects to communicate using indirect connections: Object ${ }_{i} \longrightarrow$ Server $\longrightarrow$ Object $_{j}$.

\section{Cooperating site architecture}

The site architecture as depicted by Fig. 6 is designed following a three-layers model composed of:

- an upper layer called the cooperation layer in charge of the production, the exchange and the update of the collaborative information.

- a medium layer called a coordination layer in charge of the management of the collaborative activity; and

- a lower layer called the communication layer in charge of transporting collaborative information and coordination statements.

The (group-)communication layer is composed of the Communication Representative. The Coordination Representative is the main component of the coordination layer. The cooperation layer contains the cooperating objects.

\section{The cooperating objects.}

Cooperating Objects. A cooperating object is a CORBA object. It implements an IDL interface CooperatingObject. A cooperating object is indicated by a group number represented by the attribute groupId. The behavior of an object is determined by the attribute typeId. These two attributes are assigned at object creation and are unchanged during the object life cycle.

The method initialize() is called once the object created and its attributes initialized. The method kill(), is called to remove an object. 


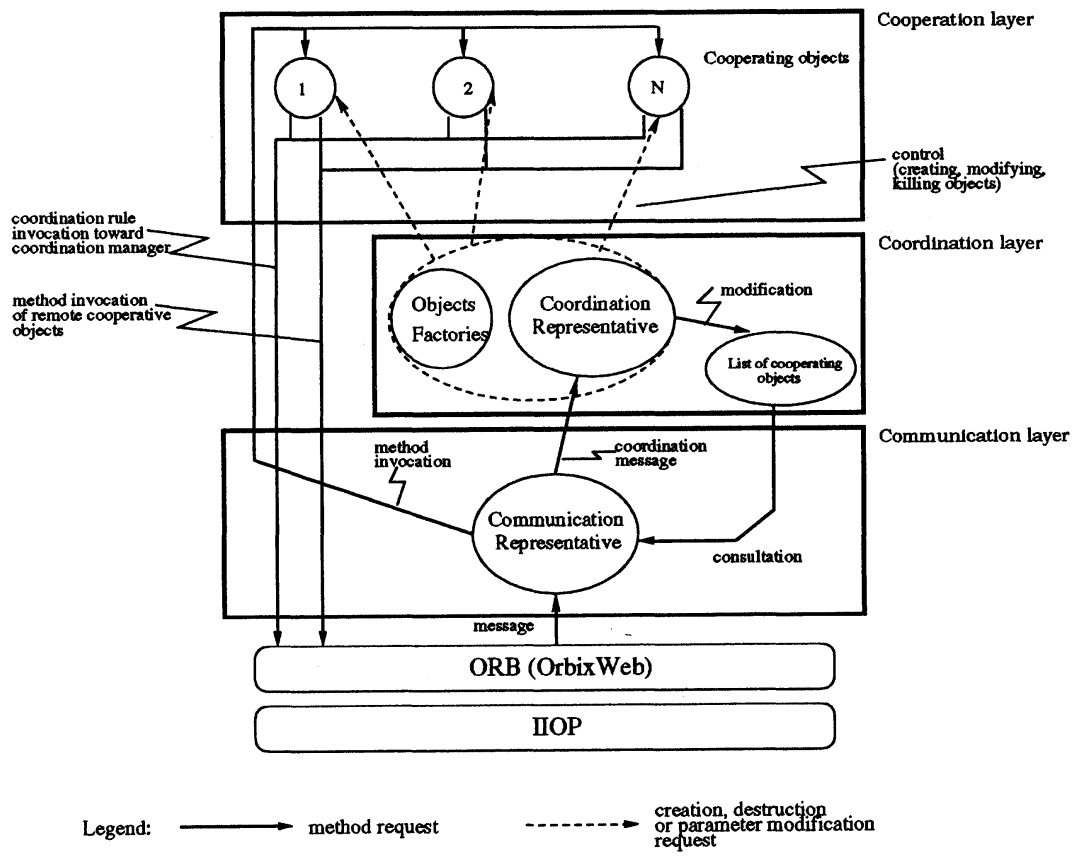

Figure 6 Cooperating Site Architecture

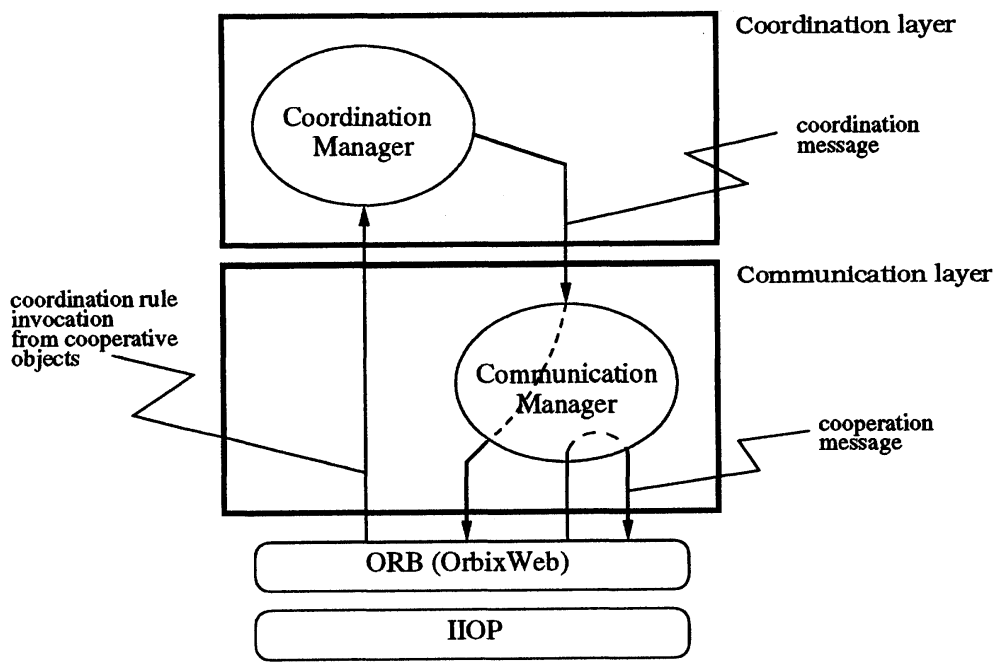

Figure 7 The coordination medium architecture 
To exchange cooperation information, the method method is used. Every cooperating object may invoke this method on every other cooperating object. The parameter methodIndex allows the invocation request to be processed by the appropriate object implementation method. The second parameter of type Any allows the programmer to specify the type of the exchanged cooperation information.

An object has two parameters. The first is the groupParameter. Its value is shared all objects belonging to a same group. This value is modified only by an application of coordination rules. The second, ownParameter, may change from an object to another. Its value is handled by the object itself without any coordination action.

Another attribute of cooperating objects is called siteRef. It is initialized at object creation and allows the object to access to different services necessary to its activity: the invocation of methods on other cooperating objects groups and the invocation of coordination rules.

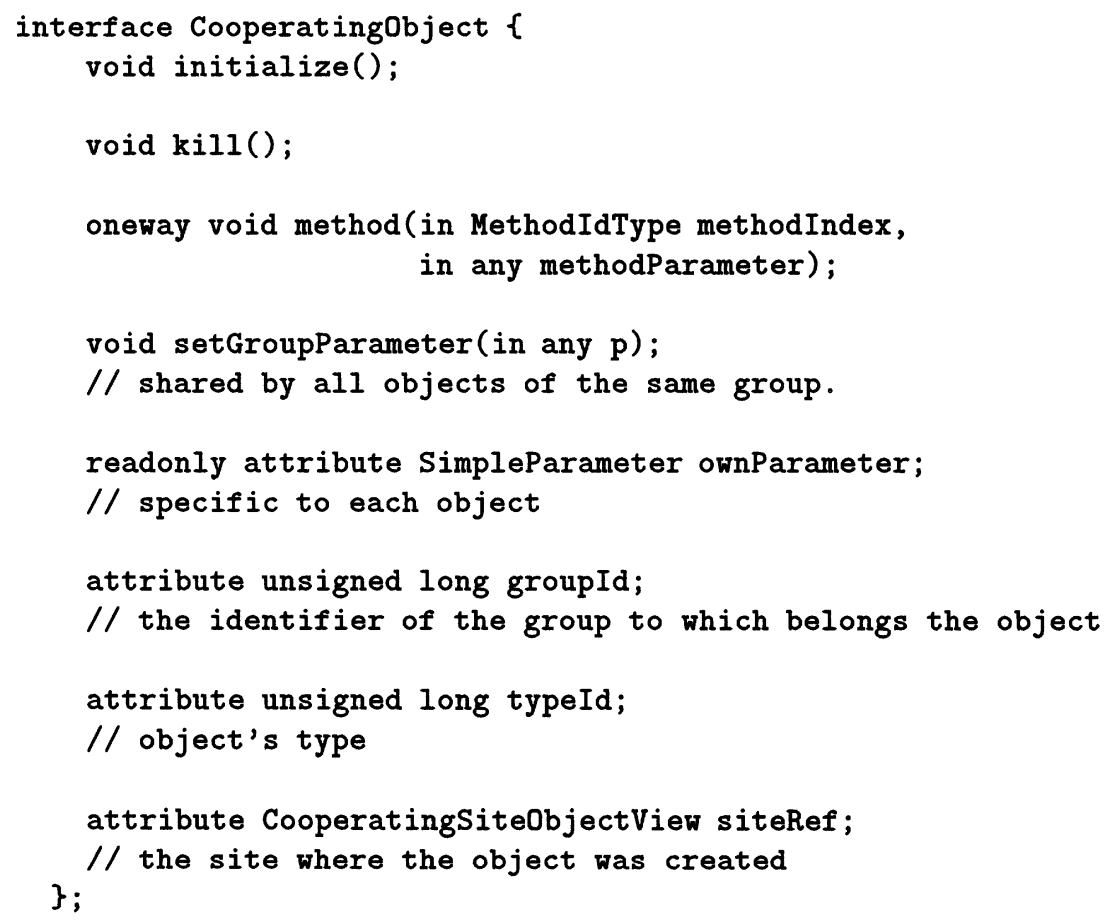

The Communication Representative. The Communication Representative is a CORBA object that processes the callbacks coming from the Communication Manager in response to remote call of methods by cooperating objects. Two types of messages may be received. Coordination messages are forwarded 
to the Coordination Representative. Cooperation messages are converted into method invocation on a group of addressed objects.

The Coordination Representative. The Coordination Representative is a CORBA object which is in charge of processing coordination messages generated by the Coordination Manager. A coordination message is a set of statements allowing creating, deleting, and updating cooperating objects located on the same site as the Coordination Representative. The creation of objects is delegated to the appropriate factory. A factory is provided for each object type.

\section{The Coordination Medium Architecture}

The Coordination Medium is implemented by two CORBA objects (Fig. 7): the Communication Manager and the Coordination Manager.

The Communication Manager. This CORBA object implements a group communication protocol allowing:

- cooperation information to be exchanged within objects groups. Object address filtering considers object types, group numbers, and object parameters.

- coordination actions to be sent to all connected sites.

The Coordination Manager. The Coordination Manager is a CORBA object in charge of managing the coordination medium implemented as a coordination graph accessible by coordination rules. Enabling a rule is done by invoking the "apply" method of this object having as arguments the identifier of the rule and its parameter. When the rule is applicable, its application leads to the rewrite of the coordination graph and the derived configuration management actions are sent to the Communication Manager in order to be forwarded to all connected sites.

\section{COORDINATION RULES FOR LINEAR INFORMATION SPACE}

Several coordination services may be provided for different cooperative applications depending on how information is organized. The organization may correspond to physical ordering of information such as string precedence in a text file. It may also correspond to logical dependencies between information items.

One coordination problem consists in managing the access to different regions of the information space. We propose here to describe this coordination for linearly ordered information space.

We will show that for linearly ordered information space, information access may be coordinated by a set of four zone allocation rules as depicted in Fig. 8 where a node may be split into one, two or three nodes according to the position of the requested zone relatively to the embedding free zone. Zone releasing rules are symmetric to allocation rules. They are described in Fig. 9. When we 


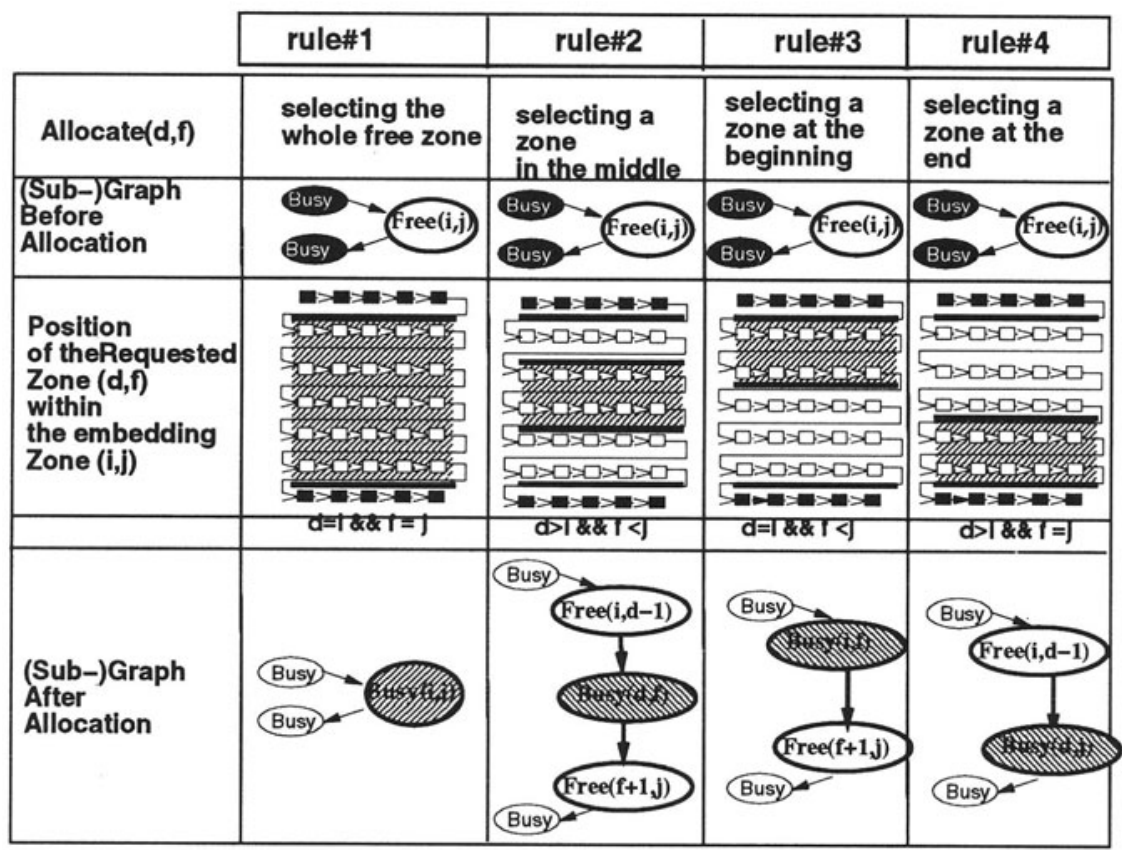

Figure 8 Coordination laws for zone allocation in linearly ordered information space

assume that modifications inside the allocated zone modify the global information ordering (such as text insertion or deletion for simultaneous text editing), an additional rule is needed to update the information ordering. The explanation of this rule needs more background on our approach for graph rewrite. It is not presented here.

The allocation rule rule\#1 describes the case where the allocated zone takes up entirely a free zone. This zone is then converted into a busy zone (the one being allocated).

The allocation rule rule\#2 describes the case where the allocated zone takes place strictly inside a free zone. This zone is then split into three new zones : a free zone, a busy zone (the one being allocated) and a free zone.

The allocation rule rule\#3 describes the case where the allocated zone is at the beginning of a free zone. This zone is then split into two new zones : a busy zone (the one being allocated) and a free zone.

The allocation rule rule\#4 describes the case where the allocated zone is at the end of a free zone. This zone is then split into two new zones : a free zone and a busy zone (the one being allocated). 


\begin{tabular}{|c|c|c|c|c|}
\hline & rule\#1' & rule\#2' & rule\#3' & rule\#4' \\
\hline Deallocate(d,f) & $\begin{array}{l}\text { releazed zone } \\
\text { Is between } \\
\text { two busy zones }\end{array}$ & $\begin{array}{l}\text { releazed zone } \\
\text { is between } \\
\text { two free zones }\end{array}$ & $\begin{array}{l}\text { releazed zone Is } \\
\text { before a free } \\
\text { zone and after } \\
\text { a busy zone }\end{array}$ & $\begin{array}{l}\text { releazed zone Is } \\
\text { before a free } \\
\text { zone and after } \\
\text { a free zone }\end{array}$ \\
\hline \multicolumn{5}{|l|}{$\begin{array}{l}\text { (Sub-)Graph } \\
\text { Before } \\
\text { Deallocation }\end{array}$} \\
\hline \multirow{6}{*}{$\begin{array}{l}\text { Position } \\
\text { of theRequested } \\
\text { Zone (d,f) } \\
\text { within } \\
\text { the embedding } \\
\text { Zone }(I, J)\end{array}$} & 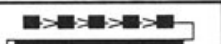 & 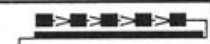 & 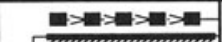 & 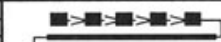 \\
\hline & 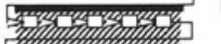 & $\Rightarrow \square>\square \geqslant \square>\square>\square$ & 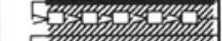 & $5 \square>\square=\square \square \square \square$ \\
\hline & & 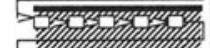 & Hanty & $\square>\square \geqslant \square>\square>\square$ \\
\hline & Stym & Otym & $\Rightarrow \square \geq \square \geq \square>\square>\square$ & $\square>\square>\square>\square>\square$ \\
\hline & 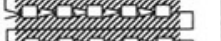 & 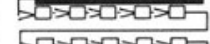 & $5 \times \square=\square>\square>\square$ & 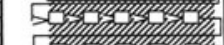 \\
\hline & Dam & 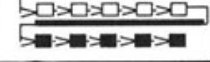 & 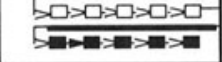 & 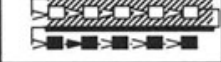 \\
\hline $\begin{array}{l}\text { (Sub-)Graph } \\
\text { After } \\
\text { Deallocation }\end{array}$ & & & & \\
\hline
\end{tabular}

Figure 9 Coordination laws for zone Deallocation in linearly ordered information space

Correctness of the coordination protocol

\section{Proposition 1 (completeness property)}

It is always possible to allocate a zone within a free zone.

To show this property, we must show that every allocation request is processed by one of the four rules constituting the coordination protocol.

Since rules allow allocation whenever the subgraph

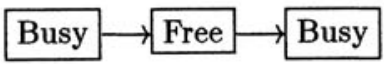

is found, the only bad situation would be a situation of the form

$$
\text { Free } \rightarrow \text { Free }
$$

indicating that the free zone exists but is preceded or followed by another free zone.

Showing the correctness is then equivalent to showing the following property.

\section{Proposition 2 (consistency property)}

The coordination graph never contain the chain

$$
\text { Free } \longrightarrow \text { Free }
$$


sketch of the proof: Assuming that the initial graph satisfies this property, we show by induction that since the rewrite rules never insert such a chain, then the coordination graph always satisfies the consistency property.

Initial graph:. In order to make the above set of four rules sufficient for solving every possible allocation request, we have to use, as initial graph, a graph that matches the common subgraph needed by these rules, i.e. a graph of the form:

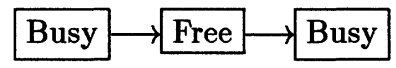

Assuming that information items are numbered using positive integers, we use two virtual busy zones whose parameters are negative numbers. If we have initially $n$ items, the initial graph may be:

$$
\operatorname{Busy}(-1,-1) \rightarrow \operatorname{Free}(1, \mathrm{n}) \rightarrow \operatorname{Busy}(-1-1)
$$

Application: structure-independent multi-user text editor

The sedit application is a multi-user text editing tool whose interface was implemented using Java 's graphical libraries. No structuration hypothesis is required to manage the text sharing. The users may request access in read/write mode within a specific window whose coordinates are specified by the user by selecting the text she/he wants to access to. This event is associated with the set of allocation rules. Application of rules is sequentially requested until one of them is applicable. When a solution is possible, a new window is allocated inside the user's interface and the text color of the allocated window changes in the main windows of remote participants, indicating the limits of the allocated window.

The transfer of text from the Read/Write window to the main window is called update. The updated text is simultaneously transmitted to all users and interfaces of these users are updated.

The release of the windows implies the destruction of the window and freeing the allocated zone.

The sedit application coordination rules are implemented as an instance of the linearly ordered information space coordination rules. The text is considered as a chain of windows that may be in one of two states: Free or Busy. Access in read-only is possible to any participant. Access in read/write mode is managed by opening limited read-write access windows for the allocated zone.

\section{COMPARISON WITH GRAPH-BASED COORDINATION TECHNIQUES}

The coordination task is essential in concurrent and distributed activities $[11,2]$. This task is particularly important for workflow management and distributed cooperative work. Two main categories of coordination tasks may be distin- 
guished within this area: the configuration management and the activity management. The former is common to all applications and deals with multi-site coordination and components creation and destruction. The latter, is specific to applications and deals with enabling and controlling the cooperation of a group of persons performing a common work.

\section{Graph transformation for activity management}

Graph rewriting techniques were used to model coordination in concurrent activities. A typical solved problem is the dining philosophers problem as in [8]. Graph rewriting is also proposed as a semantics for concurrent languages. In [8], the semantics of actor languages is described in $\Delta$-grammar. In [9] a graph rewriting semantics is developed for process calculi. In this technique graphs are an alternative syntax for process calculi. Nodes and arcs represent processes and channels. Coordination is entirely described using communication.

\section{Graph transformation for configuration management}

The configuration management constitutes an active research area. Several models and languages are proposed for coordination [6, 7].

A graph-based coordination description technique is defined in [12]. This technique allows dynamic evolution of configuration to be described using conditional graph rewrite rules. The description language uses Processes to describe the behavior of coordinated components. This work shows the importance of such formally defined techniques for correctness verification of the described systems. Nevertheless, using context-free graph grammars makes some coordination problems hard, and sometimes impossible, to describe.

\section{Graph transformation for activity and configuration management}

Like [12] and [9], we choose graphs as a tool to describe coordination for two reasons. First because of friendliness, second to provide a general technique independent of any implementation language. Describing the graph evolution using graph-grammar productions provides a formal basis for verification of coordination protocol descriptions. The graph grammar we use allows a large class of coordination problems to be described.

\section{CONCLUSION}

This paper proposed a coordination technique based on graph description and transformation technique and its application to distributed objects using COR$\mathrm{BA}$. This coordination technique provides a general approach allowing the users to specify (in a rule-oriented style) how to enable and to control both the cooperation activity and the appropriate architecture dynamic evolution. The originality and the interest of this work come from a number of advantages. First, it allows coordination protocols to be described independently from the cooperation paradigms underlying the model or the language used by the co- 
ordinated objects to cooperate. Second, the graph structure is a very general abstract structure which is appropriate to represent numerous coordination schemas. Moreover proofs may very clearly be established to validate the coordination protocols described according to this technique.

The presented coordination technique was implemented using the Java programming language and OrbixWeb ORB. The developed environment is constituted of a hundred of classes corresponding to one Megabyte Java source code. We have recently added new IDL definitions and $\mathrm{C}++$ classes allowing cooperative object behavior to be described in $\mathrm{C}++$.

The cooperative object graph transformation environment is the kernel of a coordination environment we have developed and used to implement coordination protocols for a number of existing cooperation tools and new cooperation tools. Our first utilization of the coordination environment for building the text sharing application (http://www.laas.fr/“gouezec/editor) demonstrated that such rule-oriented programming technique is a very promising technique for distributed cooperative applications development and component integration. The application-specific code (including coordination rules and cooperating objects behaviors) corresponds only to $33 \%$ of the whole application size.

The current service architecture allows different coordination medium to be created but they must share the same coordination rule set. These rules are specified at compilation time by the programmer. The server side has to be shut down to modify coordination protocols.

We are currently working on the definition and the implementation of an improved CORBA coordination service allowing coordination rules to be described and to be added at run-time. This would allow different coordination media with different coordination rules to be created. Moreover, each medium may be transformed by deleting some rules or by adding new rules allowing better evolutivity of applications.

In addition the architecture should allow generic coordination protocols to be provided as CORBA services that applications may use. In this case, applications will dynamically specify on one hand, the coordination rules that should be applied for each coordination events and, on the other hand, the management actions associated with the coordination rules.

\section{References}

[1] R.M. Adler. Distributed coordination models for client/server computing. IEEE Computer, 28(4):14-22, April 1995.

[2] S. Aggarwal, D. Barbará, and K.Z. Meth. A software environment for the specification and analysis of problems of coordination and concurrency. IEEE Transactions on Software Engineering, 14(3):280-290, March 1988.

[3] G. Agha, P. Wegner, and A. Yonezawa, editors. Research Directions in Concurrent Object-Oriented Programming. MIT Press, 1993. 
[4] J.P. Banatre and D. Le Metayer. Programming by multiset transformation. Communications of the ACM, 36(1):98-111, January 1993.

[5] N. Carriero and D. Gelernter. Generative communication in Linda. Communications of the ACM, 32(4):444-458, 1989.

[6] P. Ciancarini. Coordination Models and Languages as Software Integrators. ACM computing Surveys, 26(1):87-119, 1994.

[7] P. Ciancarini and C. Hankin, editors. Coordination Languages and Models, volume 1061 of Lecture Notes in Computer Science. Springer Verlag, 1996.

[8] S. Kaplan, J.P. Loyall, and S.K. Goering. Specifying Concurrent Languages and Systems with $\Delta$-Grammars. In Agha et al. [3].

[9] B. König and B. Reichel. Graduiertenkolleg Kooperation und Ressourcenmanagement in verteilten Systemen. Technical Report TUM-I9707, Technische Universität München, 1997.

[10] T.G. Lewis. Where is client/server software headed? IEEE Computer, 28(4):49-55, April 1995.

[11] T.W. Malone and K. Crwoston. The interdisciplinary Study of Coordination. ACM computing Surveys, 26(1):87-119, 1994.

[12] D. Le Métayer. Software architectures styles as graph grammars. In ACM SIGSOFT Symposium on the Foundations of Software Engineering, CA, USA, 1996. ACM.

[13] Thomas J. Mowbray and Ron Zahavi. The Essential CORBA: Systems Integration Using Distributed Objects. OMG/Wiley (ISBN 0-471-106119), 1995. http: //www.omg.org/tombook.htm.

[14] Christophe Gransart Philippe Merle, Jean-Francois Roos, and Jean-Marc Geib. Corbascript: A dedicated corba scripting language. In CHEP'98 Computing in High Energy Physics, Chicago, Illinois, USA, August 31 September 4, 1998.

[15] P. Wegner. Tradeoffs between Reasoning and Modeling. In Agha et al. [3]. 